Copyright 2007 Society of Photo-Optical Instrumentation Engineers. One print or electronic copy may be made for personal use only. Systematic reproduction and distribution, duplication of any material in this paper for a fee or for commercial purposes, or modification of the content of the paper are prohibited.

\title{
Fast and Accurate Border Detection in Dermoscopy Images Using Statistical Region Merging
}

\author{
M. Emre Celebi; Hassan A. Kingravi; Hitoshi Iyatomi; \\ JeongKyu Lee; Y. Alp Aslandogan; William Van \\ Stoecker; Randy Moss; Joseph M. Malters; Ashfaq A. \\ Marghoob
}

M. Emre Celebi ; Hassan A. Kingravi ; Hitoshi Iyatomi ; JeongKyu Lee ; Y. Alp Aslandogan, et al. "Fast and Accurate Border Detection in Dermoscopy Images Using Statistical Region Merging", Proc. SPIE 6512, Medical Imaging 2007: Image Processing, 65123V (March 07, 2007);

doi:10.1117/12.709073

http://dx.doi.org/10.1117/12.709073 


\title{
Fast and Accurate Border Detection in Dermoscopy Images Using Statistical Region Merging
}

\author{
M. Emre Celebi* and Hassan A. Kingravi \\ Dept. of Computer Science \& Engineering \\ University of Texas at Arlington \\ Arlington, TX, USA \\ JeongKyu Lee \\ Dept. of Computer Science \& Engineering \\ University of Bridgeport \\ Bridgeport, CT, USA
}

William Van Stoecker

Department of Computer Science

University of Missouri, Rolla Rolla, MO, USA

Joseph M. Malters

The Dermatology Center

Rolla, MO, USA

\author{
Hitoshi Iyatomi \\ Dept. of Electrical Informatics \\ Hosei University \\ Tokyo, Japan \\ Y. Alp Aslandogan \\ Dept. of Computer Science \\ Prairie View A\&M University \\ Prairie View, TX, USA \\ Randy Moss \\ Dept. of Electrical \& Computer Engr. \\ University of Missouri, Rolla \\ Rolla, MO, USA
}

\author{
Ashfaq A. Marghoob \\ Mem. Sloan-Kettering Skin Cancer Ctr. \\ Hauppauge, NY, USA
}

\begin{abstract}
As a result of advances in skin imaging technology and the development of suitable image processing techniques during the last decade, there has been a significant increase of interest in the computer-aided diagnosis of melanoma. Automated border detection is one of the most important steps in this procedure, since the accuracy of the subsequent steps crucially depends on it. In this paper, a fast and unsupervised approach to border detection in dermoscopy images of pigmented skin lesions based on the Statistical Region Merging algorithm is presented. The method is tested on a set of 90 dermoscopy images. The border detection error is quantified by a metric in which a set of dermatologist-determined borders is used as the ground-truth. The proposed method is compared to six state-of-the-art automated methods (optimized histogram thresholding, orientation-sensitive fuzzy c-means, gradient vector flow snakes, dermatologist-like tumor extraction algorithm, meanshift clustering, and the modified $J S E G$ method) and borders determined by a second dermatologist. The results demonstrate that the presented method achieves both fast and accurate border detection in dermoscopy images.
\end{abstract}

Keywords: Computer-Aided Diagnosis, Skin Lesion, Melanoma, Dermoscopy, Segmentation, Border Detection, Statistical Region Merging

\section{Introduction}

Malignant melanoma has consistently had one of the most rapidly increasing incidence of all cancers, with 62,190 new cases and 7,700 deaths estimated in the United States in 2006 [1]. Early diagnosis is particularly important since melanoma can be cured with a simple excision if detected early.

${ }^{*}$ Corresponding author email: celebie@umr.edu 
Dermoscopy is a non-invasive skin imaging technique that uses optical magnification and either liquid immersion and low angle-of-incidence lighting or cross-polarized lighting to make the contact area translucent, making subsurface structures more easily visible when compared to conventional macroscopic (clinical) images. Dermoscopy allows the identification of dozens of morphological features such as pigment networks, dots/globules, streaks, blue-white areas, and blotches [2]. This reduces screening errors, and provides greater differentiation between difficult lesions such as pigmented Spitz nevi and small, clinically equivocal lesions [3]. However, it has been demonstrated that dermoscopy may actually lower the diagnostic accuracy in the hands of inexperienced dermatologists [4]. Therefore, due to the lack of reproducibility and subjectivity of human interpretation, the development of computerized image analysis techniques is of paramount importance [5].

The first step in the computerized analysis of skin lesion images is the detection of the lesion borders. The importance of border detection for the analysis is two-fold. First, the border structure provides important information for accurate diagnosis. Many clinical features such as asymmetry, border irregularity, and abrupt border cutoff are calculated from the border. Second, the extraction of other important clinical features such as atypical pigment networks, globules, and blue-white areas critically depends on the accuracy of border detection.

Automated border detection in dermoscopy images is a challenging task due to several reasons: $i$ ) low contrast between the lesion and the surrounding skin, ii) irregular and fuzzy lesion borders, iii) artifacts such as skin texture, air bubbles and hairs, and $i v$ ) variegated coloring inside the lesion.

In this paper, an unsupervised approach to border detection in dermoscopy images based on the Statistical Region Merging $(S R M)$ algorithm [6,7] is presented. The $S R M$ algorithm is adapted to this problem due to its simplicity, computational efficiency, and excellent performance on a variety of image domains.

The rest of the paper is organized as follows: Section 2 presents the related work. Section 3 delineates the preprocessing steps that facilitate the border detection procedure. Section 4 briefly describes the $S R M$ algorithm. Section 5 details the postprocessing steps that yield the final lesion border. Section 6 discusses the experimental results and compares the proposed method with six alternative approaches. Finally, Section 7 gives the conclusions and directions for future research.

\section{Related Work}

Numerous methods have been developed for border detection in pigmented skin lesion images most of which deal with clinical images [8]. However, recent research has focused more on dermoscopy images. This section briefly reviews the border detection methods applied to these images.

Gao et al [9] proposed two methods: one based on stabilized inverse diffusion equations (SIDE), a form of non-linear diffusion and another one based on Markov Random Fields $(M R F)$ in which the model parameters are estimated using the mean field theory. Pagadala [10] described a method based on optimized histogram thresholding. Schmid [8] developed a technique based on color clustering. First, a $2 D$ histogram is calculated from the first two principal components of the $C I E L^{*} u^{*} v^{*}$ color space. The histogram is then smoothed and initial cluster centers are determined from the peaks using a perceptron classifier. Finally, the lesion image is segmented using a modified version of the fuzzy c-means $(F C M)$ clustering algorithm. Donadey et al [11] presented a supervised method based on intensity radial profiles calculated from the I (intensity) component of the HSI space. Haeghen et al. [12] proposed a very simple supervised method that exploits the CIE $L^{*} a^{*} b^{*}$ color space. In this method, the background skin color is determined from a rough outer border drawn by the user. This initial border is then shrunk as long as the local color is similar to the background skin color. Cucchiara et al [13] presented a recursive $F C M$ clustering technique that augments Schmid's method using topological information. Erkol et al [14] proposed a method based on the gradient vector flow $(G V F)$ snakes [15] with an automatic initialization. Iyatomi et al [16] described a method called the dermatologist-like tumor extraction algorithm (DTEA) that is based on thresholding followed by iterative region growing. Melli et al [17] compared four different color clustering algorithms: median cut, k-means, FCM, and meanshift [18]. They concluded that the meanshift algorithm gave the best results. Celebi et al [19] developed a method based on the JSEG segmentation algorithm [20]. Their method involves an algorithm for approximate lesion localization that reduces the computational time and improves the accuracy by focusing the border detection process on the immediate neighborhood of the lesion rather than the whole image. 


\section{Preprocessing}

This section describes the preprocessing steps that facilitate the border detection procedure, namely the black frame removal and image smoothing.

\subsection{Black Frame Removal}

Dermoscopy images often contain black frames that are introduced during the digitization process. These need to be removed because they might interfere with the subsequent border detection steps. In order to determine the darkness of a pixel with $(R, G, B)$ coordinates, the lightness component of the $H S L$ color space [21] is utilized:

$$
L=\frac{\max (R, G, B)+\min (R, G, B)}{2}
$$

In particular, a pixel is considered to be black if its lightness value is less than 20. Using this criterion, the image is scanned row-by-row starting from the top. A particular row is labeled as part of the black frame if it contains $60 \%$ black pixels. The top-to-bottom scan terminates when a row that contains less than the threshold percentage of pixels is encountered. The same scanning procedure is repeated for the other three main directions. Figure 1 shows the result of this procedure on a sample dermoscopy image.

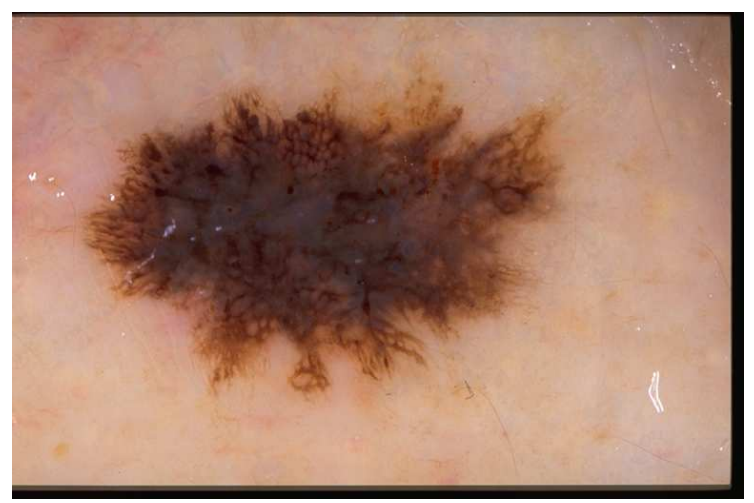

(a) Dermoscopy image with a black frame

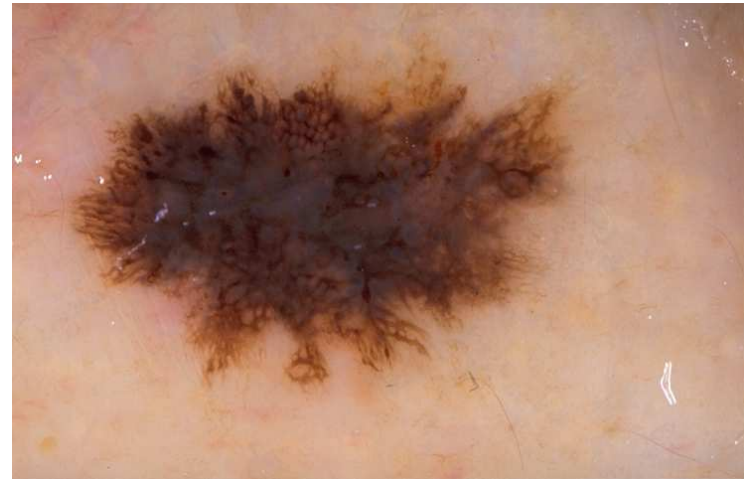

(b) Image a) after frame removal

Figure 1: Frame Removal

\subsection{Image Smoothing}

Dermoscopy images often contain extraneous artifacts such as skin lines, air bubbles and hairs around the lesion. These might reduce the accuracy of the border detection and increase the computational time. In order to mitigate the detrimental effects of these artifacts, the images should be preprocessed with a smoothing filter.

The median filter is one of the most common smoothing filters in the literature [22]. Median filtering with a mask of appropriate size can eliminate most of the artifacts in a dermoscopy image [8]. Figure 2 shows the result of channel-wise median filtering on a sample image. It can be seen that this operation is very effective in reducing the thin hairs and air bubbles.

Note that the mask size should be proportional to the image size for optimal results. In this study, given an $M$ by $N$ image, the mask size $n$ is determined by:

$$
n=\lfloor 5 \sqrt{(M / 768)(N / 512)}\rfloor
$$

Equation (2) is based on the observation that for a typical $768 \times 512$ image $n=5$ is a good choice and it ensures that when the image size changes, this is reflected on the mask size proportionally. 


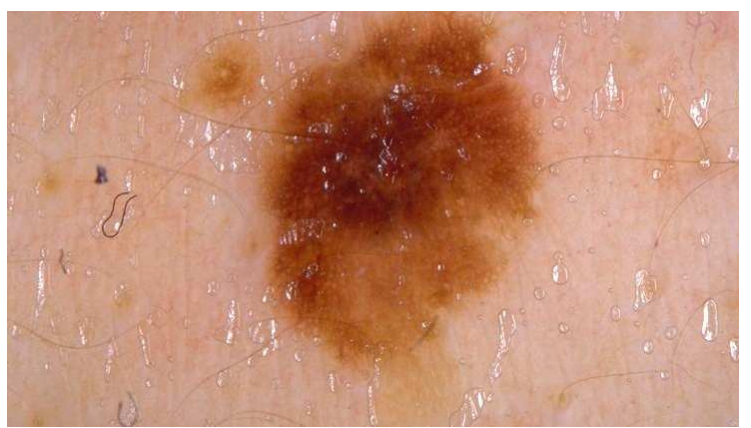

(a) Dermoscopy image with bubbles and hair

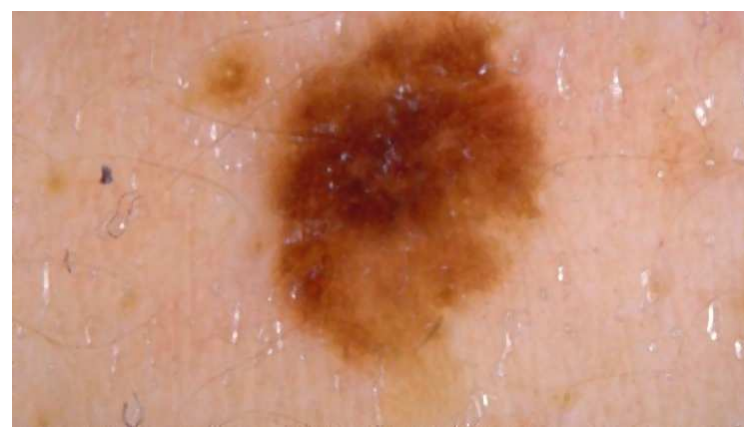

(b) Image a) after median filtering

Figure 2: Median Filtering

\section{Statistical Region Merging}

Statistical Region Merging $(S R M)$ is a recent color image segmentation technique based on region growing and merging. The method models segmentation as an inference problem, in which the image is treated as an observed instance of an unknown theoretical image, whose statistical (true) regions are to be reconstructed. The advantages of this method include its simplicity, computational efficiency, and excellent performance without the use of quantization or color space transformations.

Let $I$ be an observed image that contains $|I|$ pixels, each of which is comprised of $R, G, B$ color channel values belonging to the set $\{0,1, \ldots, g-1\}$ (where $g=256$ for 8 bit $R G B$ images). $I$ is an observation of a true image $I^{*}$ in which pixels are perfectly represented by a family of distributions from which each of the observed color channel is sampled. The optimal statistical regions in $I^{*}$ share a homogeneity property such that inside any statistical region and given any color channel, the statistical pixels have the same expectation, whereas the expectations of adjacent statistical regions differ in at least one color channel.

$I$ is obtained from $I^{*}$ by sampling each statistical pixel for observed $R G B$ values. The color channel values for every pixel in $I^{*}$ are replaced by a set of $Q$ independent random variables, which take on values from $[0, g / Q]$. It is to be noted that the $Q$ parameter can be used to quantify the statistical complexity of $I^{*}$, the generality of the model, and the statistical difficulty of the problem. Higher values of $Q$ result in finer segmentation and thus the generation of more regions.

Like other region growing algorithms, $S R M$ is based on two major components; a merging predicate and the order followed in testing this predicate. The predicate is defined as:

$$
\begin{gathered}
P\left(R, R^{\prime}\right)=\left\{\begin{array}{l}
\text { true } \text { if } \forall a \in\{\mathbf{R}, \mathbf{G}, \mathbf{B}\},\left|\bar{R}_{a}^{\prime}-\bar{R}_{a}\right| \leq\left|\sqrt{b^{2}(R)+b^{2} R^{\prime}}\right| \\
\text { false } \text { otherwise }
\end{array}\right. \\
b(R)=g \sqrt{\frac{1}{2 Q|R|} \ln \left(6|I|^{2} R_{|R|}\right)}
\end{gathered}
$$

where $R$ and $R^{\prime}$ represent the two regions being tested, $\bar{R}_{a}$ denotes the observed average for color channel $a$ in region $R$ and $R_{|p|}$ is the set of regions with $p$ pixels. The order of region merging follows an invariant $A$, which implies that when any test between two parts within a true region is performed, no further tests are necessary.

Let $S_{I}$ be a set that contains all pairs of adjacent pixels in the image based on 4-connectivity, $p$ and $p^{\prime}$ be pixels in image $I$, and $R(p)$ stand for the current region to which a pixel $p$ belongs. The $S R M$ algorithm first sorts these pairs in increasing order according to a function $f\left(p, p^{\prime}\right)$. After the sorting is completed, the order is traversed only once, performing the merging test $P\left(R(p), R\left(p^{\prime}\right)\right)$ for any pair of pixels $\left(p, p^{\prime}\right)$ for which $R(p) \neq R\left(p^{\prime}\right)$, and merging $R(p)$ and $R\left(p^{\prime}\right)$ if it returns true. The complexity of the ordering phase is $O(|I| \log (g))$ when radix sort is used with color differences as the keys. Similarly, the merging phase can be performed in linear time using an efficient union-find algorithm [23].

Since the model of image generation presented above assumes that the observed color variations within true regions should be significantly smaller than those between the regions, one way to approximate $A$ is to 
calculate the between-pixel local gradients and then compute their maximum per-channel variation in $f($.$) ,$ i.e. $f\left(p, p^{\prime}\right)=\max _{a} \in\{R, G, B\} f_{a}\left(p, p^{\prime}\right)$. The simplest choice for $f($.$) is to use the pixel channel values \left(p_{a}\right.$ and $p_{a}^{\prime}$ ) directly:

$$
f\left(p, p^{\prime}\right)=\left|p_{a}-p_{a}^{\prime}\right|
$$

Alternative methods include extensions of the kernels used in edge detection operators such as the Sobel or Roberts operators.

\section{Postprocessing}

The segmented image often contains regions that are part of the background skin. In order to eliminate these regions, the background skin color needs to be determined. In this study, the approach described in [24] is adopted. Four patches of size $20 \times 20$ pixels from the corners of the image are taken and the mean $R, G, B$ of the pixels is calculated. This mean color is taken as an estimate of the background skin color. The light colored regions, i.e. the regions whose mean color has a distance less than 60 to the background skin color, are then eliminated. In addition, the regions that touch the image frame and those with rectangular borders are discarded. The initial border detection result is obtained by removing the isolated regions and then merging the remaining regions. Figures 3 (a)-(c) illustrate this procedure.

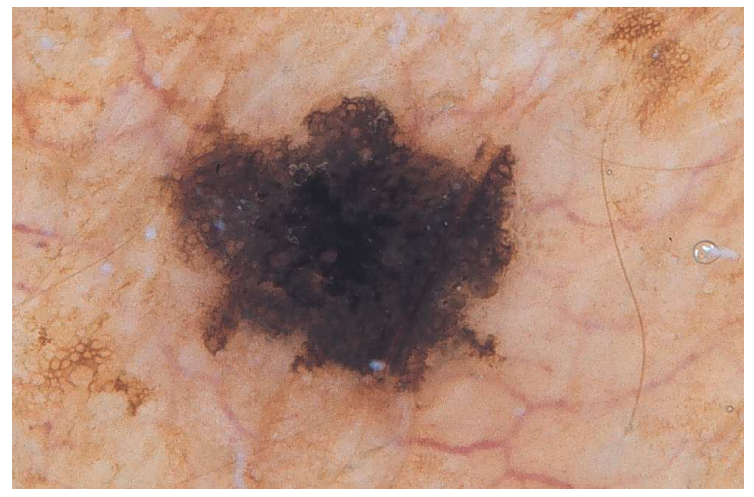

(a) Original dermoscopy image

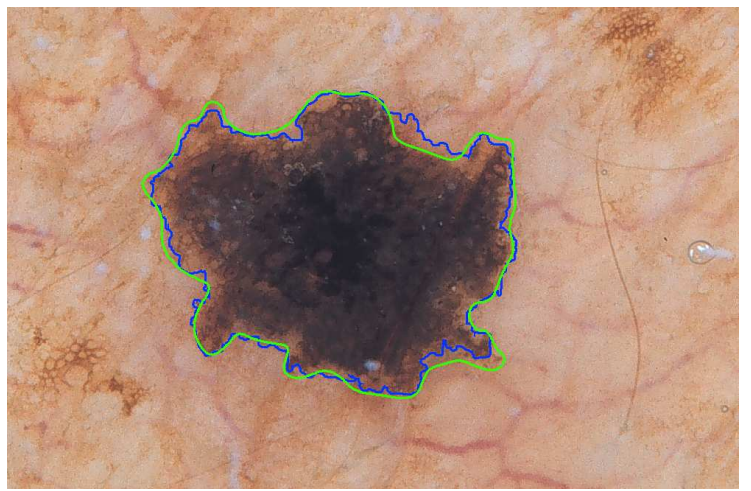

(c) Initial border detection result $\{$ Error $=8.11 \%\}$

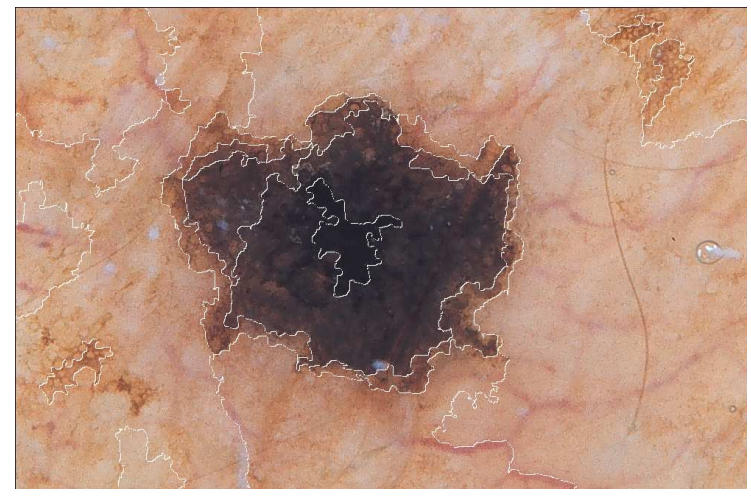

(b) Result of the $S R M$ segmentation

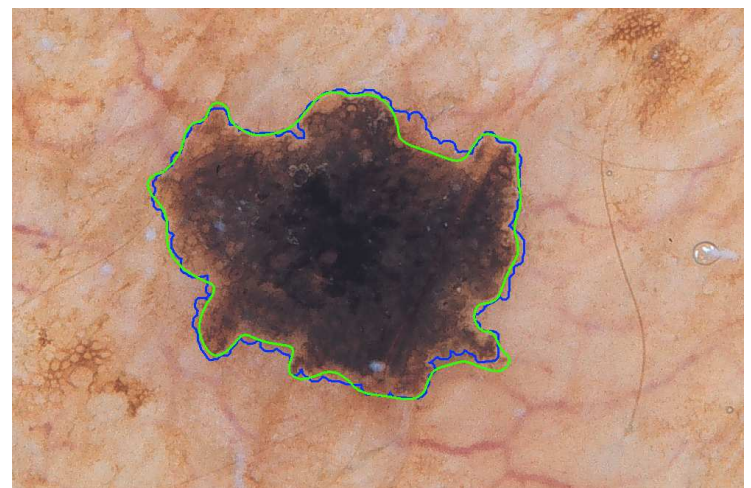

(d) Final border detection result $\{$ Error $=7.13 \%\}$

Figure 3: Illustration of the postprocessing procedure \{ green: manual border, blue: automatic border \}

Note that in Figure 3(c) the automatic border is mostly contained inside the manual border. This is observed in many cases because during the border determination procedure, the computer algorithms tend to find the sharpest pigment change, whereas the dermatologists choose the outmost detectable pigment. In order to bring the automatic border closer to the manual one, morphological dilation with a circular 
Table 1: Percentage border error statistics

\begin{tabular}{|c|c|c|c|c|c|c|}
\hline \multirow{2}{*}{ Method } & \multicolumn{2}{|c|}{ Benign } & \multicolumn{2}{c|}{ Melanoma } & \multicolumn{2}{c|}{ Overall } \\
\cline { 2 - 7 } & Mean & Stdev & Mean & Stdev & Mean & Stdev \\
\hline Second dermatologist & 8.448 & 3.747 & 7.805 & 3.489 & 8.283 & 3.674 \\
\hline Histogram thresholding & 12.546 & 7.272 & 21.332 & 15.370 & 14.791 & 10.605 \\
\hline OSFCM & 22.995 & 12.614 & 28.312 & 15.246 & 24.354 & 13.449 \\
\hline GVF snakes & 13.435 & 5.352 & 19.340 & 9.335 & 14.944 & 7.034 \\
\hline DTEA & 10.664 & 5.125 & 13.697 & 8.931 & 11.440 & 6.401 \\
\hline Meanshift & 11.526 & 9.737 & 13.291 & 7.417 & 11.977 & 9.193 \\
\hline JSEG & 10.832 & 6.358 & 13.744 & 7.590 & 11.576 & 6.772 \\
\hline SRM & $\mathbf{1 0 . 9 2 1}$ & $\mathbf{5 . 7 2 7}$ & $\mathbf{1 1 . 0 8 1}$ & $\mathbf{6 . 0 7 3}$ & $\mathbf{1 0 . 9 6 2}$ & $\mathbf{5 . 7 8 4}$ \\
\hline
\end{tabular}

structuring element of size $n=\lfloor 4(d / 500)\rfloor$ ( $d$ : diameter of the lesion) is applied to the automatic border. Figure 3(d) shows the result of this expansion. It can be seen that after the dilation, the automatic border is much closer to the manual border. The quantification of the border detection error will be explained in the next section.

\section{Experimental Results and Discussion}

The proposed method is tested on a set of 90 dermoscopy images (23 invasive malignant melanoma and 67 benign) obtained from the EDRA Interactive Atlas of Dermoscopy [25] and the dermatology practices of Dr. Ashfaq Marghoob (New York, NY), Dr. Harold Rabinovitz (Plantation, FL), and Dr. Scott Menzies (Sydney, Australia). These are 24-bit RGB color images with dimensions ranging from 577 x 397 pixels to 1921 x 1285 pixels. The benign lesions include nevocellular nevi and dysplastic nevi.

As a ground truth for the evaluation of the border detection error, manual borders are obtained by selecting a number of points on the lesion border, connecting these points by a second-order B-spline and finally filling the resulting closed curve. Two sets of manual borders are obtained by dermatologists Dr. William Stoecker and Dr. Joseph Malters using this method.

Using the borders manually determined by one of the dermatologists (Dr. Stoecker), the automatic borders obtained from the seven automated methods (optimized histogram thresholding [10], orientationsensitive fuzzy c-means $(O S F C M)$ [8], GVF snakes [14], DTEA [16], meanshift clustering [17], JSEG [19], and $S R M$ ) and the manual borders from the other dermatologist (Dr. Malters) are compared using the grading system developed by Hance et al. [26]. Here, the percentage border error is given by:

$$
\text { Border Error }=\frac{\text { Area }(\text { AutomaticBorder } \oplus \text { ManualBorder })}{\text { Area }(\text { ManualBorder })} 100 \%
$$

where AutomaticBorder is the binary image obtained by filling the computer detected border, ManualBorder is the binary image described above, $\oplus$ is the exclusive-OR operation that gives the pixels for which the AutomaticBorder and ManualBorder disagree, and Area $(I)$ denotes the number of pixels in the binary image $I$.

Table 1 gives the mean and standard deviation border error for the above-mentioned methods. It can be seen that the mean error obtained by the second dermatologist is less than those obtained by the automated methods. Among the automated methods, for the benign group, the DTEA, the JSEG and the $S R M$ methods achieve the lowest mean errors $(10.66 \%, 10.83 \%$, and $10.92 \%$, respectively). On the other hand, for the melanoma group, the $S R M$ method achieves the lowest mean error (11.08\%) followed by the meanshift (13.29\%), the DTEA (13.69\%), and the JSEG methods (13.74\%). The increased error rates in the melanoma group are possibly due to the presence of higher border irregularity and color variegation in these lesions. Overall, the $S R M$ method achieves the lowest mean error $(10.96 \%)$ among the automated methods.

An examination of the standard deviation values in Table 1 reveals that the manual borders from the second dermatologist result in error values with low standard deviation $(3.74 \%$ and $3.48 \%$ for the benign 
Table 2: Distribution of the mean border error for the automated methods over the whole image set (90 images)

\begin{tabular}{|c|c|c|c|c|}
\hline \multirow{2}{*}{ Method } & \multicolumn{4}{|c|}{ MeanBorderError } \\
\cline { 2 - 5 } & $\leq 10$ & $\leq 20$ & $\leq 30$ & $\leq 40$ \\
\hline Histogram thresholding & 38 & 69 & 82 & 88 \\
\hline OSFCM & 10 & 39 & 61 & 74 \\
\hline GVF snakes & 22 & 71 & 87 & 89 \\
\hline DTEA & 51 & 81 & 89 & 90 \\
\hline Meanshift & 48 & 83 & 86 & 89 \\
\hline JSEG & 49 & 78 & 89 & 90 \\
\hline SRM & $\mathbf{4 8}$ & $\mathbf{8 2}$ & $\mathbf{8 9}$ & $\mathbf{9 0}$ \\
\hline
\end{tabular}

and melanoma groups, respectively), indicating the high degree of consistency of the second dermatologist in border determination. Among the automated methods, for the benign group, the DTEA, the $G V F$, and the $S R M$ methods are the most consistent $(5.12 \%, 5.35 \%$, and $5.72 \%$, respectively), while for the melanoma group, the SRM method achieves the highest consistency $(6.07 \%)$ followed by the meanshift $(7.41 \%)$, and the JSEG methods (7.59\%). Overall, the $S R M$ method achieves the highest consistency (5.78\%) among the automated methods.

Table 2 shows the number of images with percentage border error less than $10 \%, 20 \%, 30 \%$, and $40 \%$ for the automated methods. It can be seen that for the SRM method, 82 of the 90 images have less than $20 \%$ border error, 89 images have less than $30 \%$ border error, and all of the images have less than $40 \%$ border error. According to this criterion, the DTEA, the meanshift, and the JSEG methods follow the proposed method closely.

Figure 4 shows sample border detection results for the $S R M$ method. The method performs well even in the presence of complicating factors such as diffuse edges, blood vessels and skin lines.

\section{Conclusions and Future Work}

In this paper, a fast and unsupervised approach to border detection in dermoscopy images based on the $S R M$ algorithm is presented. The proposed approach is comprised of three main phases: preprocessing, segmentation, and postprocessing. The preprocessing phase includes black frame removal and image smoothing. The segmentation phase includes pixel couple ordering and region merging. Finally, the postprocessing phase consists of eliminating the regions that belong to the background skin, removing the isolated regions, merging the remaining regions, and expanding the initial border by morphological dilation to obtain the final result. The execution time of the proposed method is about 0.4 seconds for a typical image of size $768 \mathrm{x} 512$ pixels on an Intel Centrino 1.6Ghz computer.

The proposed method is tested on a set of 90 dermoscopy images. A set of dermatologist-determined borders is used as the ground-truth. The border detection error is quantified by a metric developed by Hance et al. [26] computed as the number of pixels for which the automatic and manual borders disagree divided by the number of pixels in the manual border. The results are compared to six other automated methods and borders determined by a second dermatologist.

The proposed method may not perform well on images with a significant amount of hair. In such cases, parts of the detected border might follow the hairs. Figure 5 shows an example of this situation. For such images, a preprocessor that eliminates hairs such as the DullRazor [27] might be helpful.

The implementation of the SRM algorithm will be made publicly available as part of the Fourier image processing and analysis library, which can be downloaded from http://sourceforge.net/projects/fourier-ipal 




(a) Error $=5.73 \%$ (melanoma)

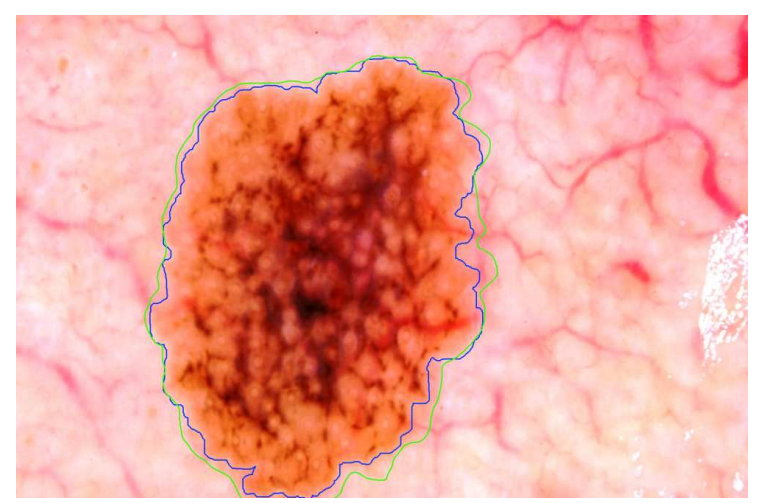

(c) Error $=8.26 \%$ (melanoma)

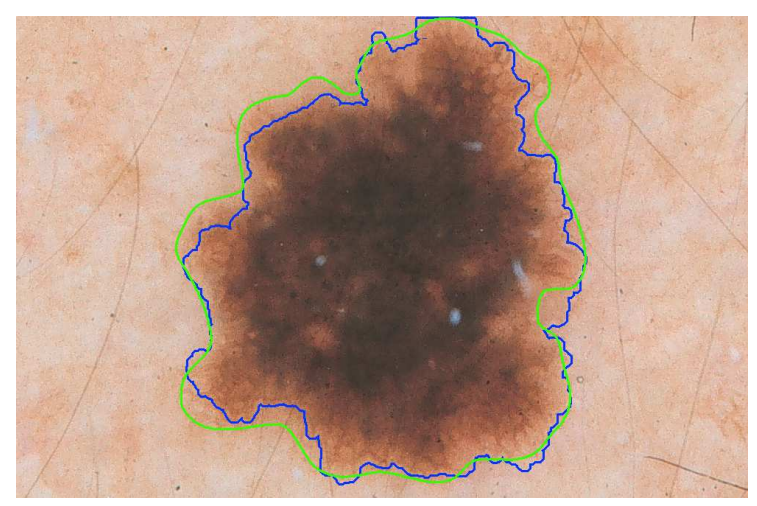

(e) Error $=11.13 \%$ (benign)

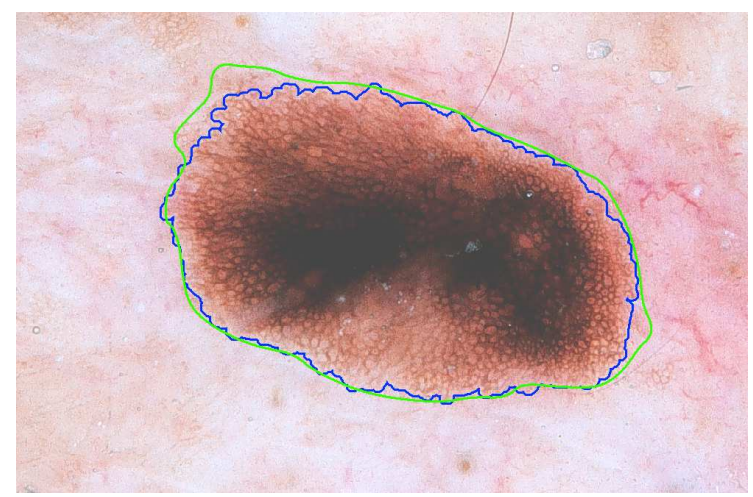

(b) Error $=7.71 \%$ (melanoma)

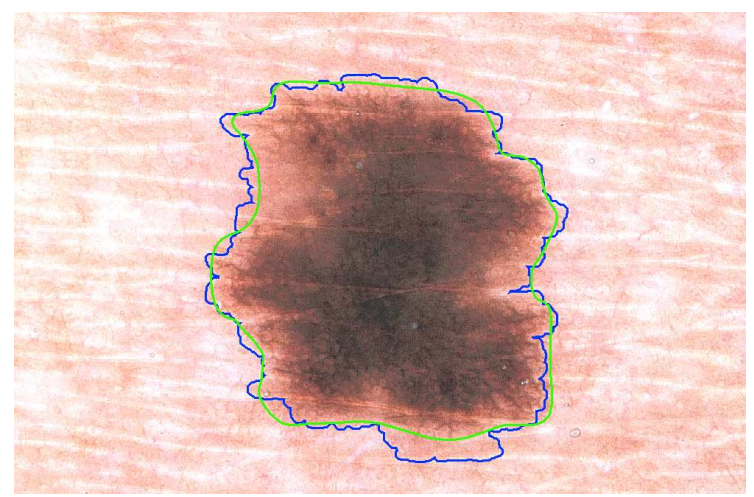

(d) Error $=9.40 \%$ (benign)

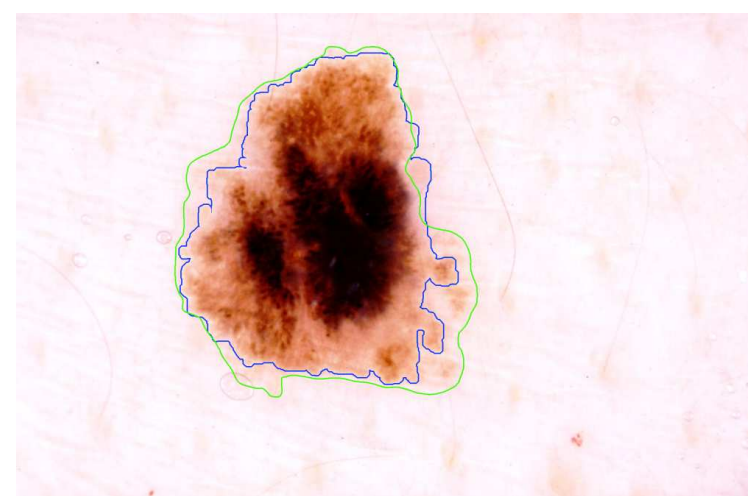

(f) Error $=17.27 \%$ (melanoma)

Figure 4: Sample border detection results for the $S R M$ method \{ green: manual border, blue: automatic border \} 


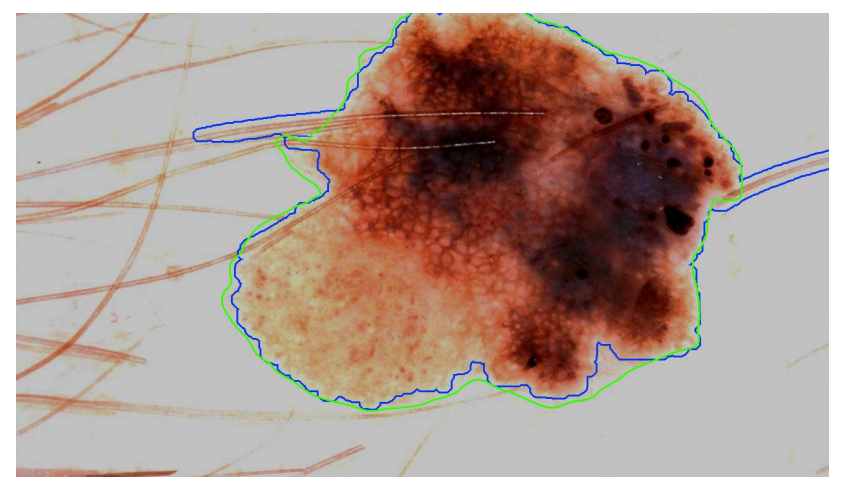

Figure 5: The effect of thick hairs on border detection \{ green: manual border, blue: automatic border \} $[$ Error $=8.12 \%]$

\section{Acknowledgements}

This work was supported by grants from NSF (\#0216500-EIA), Texas Workforce Commission (\#3204600182), James A. Schlipmann Melanoma Cancer Foundation and NIH (SBIR \#2R44 CA-101639-02A2).

\section{References}

[1] Jemal A., Siegel R., Ward E., Murray T., Xu J., Smigal C., and Thun M.J. (2006) "Cancer Statistics 2006" CA: A Cancer Journal for Clinicians, 56(1): 106-130.

[2] Menzies S.W., Crotty K.A., Ingwar C., and McCarthy W.H. (2003) An Atlas of Surface Microscopy of Pigmented Skin Lesions: Dermoscopy. Sydney, Australia: McGraw-Hill.

[3] Steiner K., Binder M., Schemper M., Wolff K., and Pehamberger H. (1993) Statistical Evaluation of Epiluminescence Dermoscopy Criteria for Melanocytic Pigmented Lesions. Journal of American Academy of Dermatology, 29(4): 581-588.

[4] Binder M., Schwarz M., Winkler A., Steiner A., Kaider A., Wolff K., and Pehamberger H. (1995) Epiluminescence Microscopy. A Useful Tool for the Diagnosis of Pigmented Skin Lesions for Formally Trained Dermatologists. Achieves of Dermatology, 131(3): 286-291.

[5] Fleming M.G., Steger C., Zhang J., Gao J., Cognetta A.B., Pollak I., and Dyer C.R. (1998) Techniques for a Structural Analysis of Dermatoscopic Imagery. Computerized Medical Imaging and Graphics, 22(5): 375-389.

[6] Nock R. and Nielsen F. (2004) Statistical Region Merging. IEEE Trans. on Pattern Analysis and Machine Intelligence, 26(11): 1452-1458.

[7] Nock R. and Nielsen F. (2005) Semi-supervised Statistical Region Refinement for Color Image Segmentation. Pattern Recognition, 38(6): 835-846.

[8] Schmid P. (1999) Segmentation of Digitized Dermatoscopic Images by Two-Dimensional Color Clustering. IEEE Trans. on Medical Imaging, 18(2): 164-171.

[9] Gao J., Zhang J., Fleming M.G., Pollak I., and Cognetta A.B. (1998) Segmentation of Dermatoscopic Images by Stabilized Inverse Diffusion Equations. Proc. of the IEEE Int. Conf. on Image Processing (ICIP'98), 3: 823-827.

[10] Pagadala P. (1998) Tumor Border Detection in Epiluminescence Microscopy Images. MS Thesis, Department of Electrical and Computer Engineering, University of Missouri- Rolla. 
[11] Donadey T., Serruys C., Giron A., Aitken G., Vignali J.-P., Triller R., and Fertil B. (2000) Boundary Detection of Black Skin Tumors Using an Adaptive Radial-based Approach. Proc. of the SPIE Medical Imaging Conf., 3379: 810-816.

[12] Haeghen Y.V., Naeyaert J.M., and Lemahieu I. (2000) Development of a Dermatological Workstation: Preliminary Results on Lesion Segmentation in CIELAB Color Space. Proc. of the First Int. Conf. on Color in Graphics and Image Processing.

[13] Cucchiara R., Grana C., Seidenari S., and Pellacani G. (2002) Exploiting Color and Topological Features for Region Segmentation with Recursive Fuzzy C-Means. Machine Graphics and Vision, 11(2/3): 169-182.

[14] Erkol B., Moss R.H., Stanley R.J., Stoecker W.V., and Hvatum E. (2005) Automatic Lesion Boundary Detection in Dermoscopy Images Using Gradient Vector Flow Snakes. Skin Research and Technology, 11(1): $17-26$.

[15] Xu C. and Prince J. L. (1998) Snakes, Shapes, and Gradient Vector Flow. IEEE Trans. on Image Processing, 7(3): 359-369.

[16] Iyatomi H., Oka H., Saito M. et al. (2006) Quantitative Assessment of Tumor Extraction from Dermoscopy Images and Evaluation of Computer-based Extraction Methods for Automatic Melanoma Diagnostic System. Melanoma Research 16(2): 183-190.

[17] Melli R., Grana C., and Cucchiara R. (2006) Comparison of Color Clustering Algorithms for Segmentation of Dermatological Images. Proc. of the SPIE Medical Imaging Conf., 6144: 3S1-9.

[18] Comaniciu D. and Meer P. (1997) Robust Analysis of Feature Spaces: Color Image Segmentation. Proc. of the IEEE Int. Conf. on Computer Vision and Pattern Recognition (CVPR'97), pp. 750-755.

[19] Celebi M.E., Aslandogan Y.A., Stoecker W.V., Iyatomi H., Oka H., and Chen X. (2006) Unsupervised Border Detection in Dermoscopy Images. accepted for publication in Skin Research and Technology

[20] Deng Y. and Manjunath B.S. (2001) Unsupervised Segmentation of Color-Texture Regions in Images and Video. IEEE Trans. on Pattern Analysis and Machine Intelligence, 23(8): 800-810.

[21] Levkowitz H. and Herman G.T. (1993) GLHS: A Generalized Lightness, Hue, and Saturation Color Model. CVGIP: Graphical Models and Image Processing, 55(4): 271-285.

[22] Umbaugh S.E. (2005) Computer Imaging: Digital Image Analysis and Processing. Boca Raton, FL: CRC Press.

[23] Fiorio C. and Gustedt J. (1996) Two Linear time Union-Find Strategies for Image Processing. Theoretical Computer Science, 154(2): 165-181.

[24] Xu L., Jackowski M., Goshtasby A., Roseman D., Bines S., Yu C., Dhawan A., and Huntley A. (1999) Segmentation of Skin Cancer Images. Image and Vision Computing, 17(1): 65-74.

[25] Argenziano G., Soyer H.P., De Giorgi V., Piccolo D., Carli P., Delfino M. et al. (2002) Dermoscopy: A Tutorial. Milan, Italy: EDRA Medical Publishing \& New Media.

[26] Hance G.A., Umbaugh S.E., Moss R.H., and Stoecker W.V. (1996) Unsupervised Color Image Segmentation with Application to Skin Tumor Borders. IEEE Engineering in Medicine and Biology Magazine, 15(1): 104-111.

[27] Lee T.K., Ng V., Gallagher R., Coldman A., and McLean D. (1997) Dullrazor: A Software Approach to Hair Removal from Images. Computers in Biology and Medicine, 27(6): 533-543. 